\title{
Studying DOZE-OFF in student using ELECTROENCEPHALOGRAPHY system
}

\author{
Le Q. Khai ${ }^{*}$, Pham T. H. Trang, Nguyen T. Hieu, Huynh T. D. Thy, Huynh Q. Linh
}

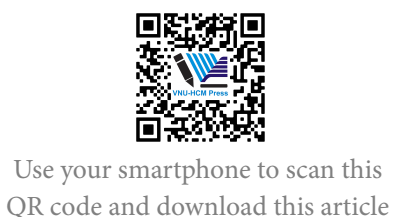

Faculty of Applied Science, Ho Chi Minh University of Technology, VNU-HCM, Vietnam

\section{Correspondence}

Le Q. Khai, Faculty of Applied Science, Ho Chi Minh University of Technology, VNU-HCM, Vietnam

Email: quockhai@hcmut.edu.vn

History

- Received: 10-02-2020

- Accepted: 24-11-2020

- Published: 15-1-2021

DOI : 10.32508/stdjet.v3iSI3.671

\section{Check for updates}

Copyright

(c) VNU-HCM Press. This is an openaccess article distributed under the terms of the Creative Commons Attribution 4.0 International license.

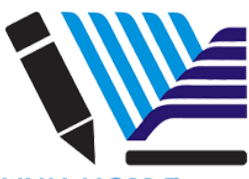

VNU-HCM Press

\begin{abstract}
Sleep deprivation of high school and university students is currently an actual concerning issue. Sleep deprivation is one of the leading causes of dozing-off during daytime. Usually, the state of drowsiness is very little concerned, but studies on drowsiness show the importance of investigating the frequency of occurrence as well as the need to clarify the cause and propose limited measures appeared dozing-off. Dozing-off is not only an undesirable state and disrupts daily activities, but also provides information on personal health status. In that case, early alertness for dozing-off event is very helpful in preventing unwanted consequences. The study has designed a process to record dozing-off event, then constructed and implemented the hypnogram processing program that evaluated quantitative changes in polysomnography signals at sleep onset, the transition time from wake stage to sleep stage. By analyzing the energy spectrum of the signal and using wavelet transform in combine with the support vector machine algorithm, the research allows a comprehensive evaluation of the state of dozing-off. Determining the exact time of onset of sleep is very important in the study of drowsiness. Extracting the time of this event appears to help develop an application for early warning dozing-off. Besides, it allows making an initial assessment of the condition of the subject when the time of drowsiness begins suddenly. Six recordings from volunteered students were processed from a 45-minute vigilance test. All of the volunteers had no neuropathy and were well explained to the procedures used in this study. The results show that depending on kinds of different applications, signals such as EEG, EOG, or EMG are used individually or in combination to fetch suitable results. The result presented a successful method to distinguish dozing-off event with other stages.

Key words: dozing-off, sleep onset, polysomnography, support vector machine
\end{abstract}

\section{INTRODUCTION}

OZING-off can be observed in many workplaces, public spots and especially in educational environment. This phenomenon causes many unwanted consequences and becomes an actual concerning issue since it leads to serious occupational accidents and reduces life quality. For students, dozing-off causes distraction from studying which reduces their productivity.

Dozing-off can be a symptom of many severe illness and disorders such as narcolepsy or sleep deprivation ${ }^{1}$. Researchers also found that people who frequently doze-off has the hazard ratio for getting stroke 2.6 times higher than normal ${ }^{2}$. Due to its harmfulness, early alertness is necessary in order to prevent people from accidents and keep their work on track. Also, a full record of dozing-off events can be useful for further diagnostic and therapy.

Reasons for daytime unwanted sleep can be listed as a side effect of medicine or fatigue due to overwork. Above all, lacking of sleep is the most mentioned rea- son when talking about dozing-off. This is also the frequent answer when asking students why they doze-off during class or when they drive to school in the morning ${ }^{3}$.

Many researches were conducted to detect this undesirable sleeping condition using infrared camera analyzing movements of the head and pupils. This device is attached to cars as an alarm for drivers and is now widely used thanks to its noninvasive function. However, there are some limitations in this device. As a peripheral equipment, it is affected by noise from outside environment which leads to errors in detection ${ }^{4}$. In addition to this, sleeping condition is controlled by the brain. Then, a detection system based on brain signals will work better at distinguish the stages. In that case, 3 special characters usually used for classifying sleep stages are Electroencephalogram (EEG), Electromyogram (EMG) and EOG (Electrooculogram) signals which are acquired from the Electroencephalography system.

Dozing-off event can be detected by determining the transition time between wake and sleep stages (or 
the sleep on-set condition) when a subject is doing some particular actions. To have a better result of the dozing-off records, a precise measurement for transition time is necessary. This work can be best achieved by the convergence of a number of indices ${ }^{5}$. In this project 3 indices were data collected from 3 channels of EEG, EMG and EOG signals.

When the transition period happens between wake and sleep stages, muscle tone presents a reduction in amplitude. Normally, in relaxation period, EEG displays a predominant of alpha activity (8 to $13 \mathrm{~Hz}$ ). When sleep enters, the theta activity ( 4 to $7 \mathrm{~Hz}$ ) replaces and usually accounts for $50 \%$ of epoch duration. For the EOG performance, during wake stage, the channels in both eyes show eye blinking and rapid eye movements. As the patient become extremely drowsy, slow eye movement appears, and sleep may come right after. That is why the presence of slow eye movement is considered to be a proof for sleep onset. When combining with other characters, the feature increases the confidence in recognizing the sleep on-set condition ${ }^{5}$.

However, when the body relaxes, muscle tone also scales down as well as in the transition time between REM and NREM sleeps. Because of that reason, EMG signal itself cannot be used alone as an indicator for sleep on-set scoring. Similarly for EEG signal, the predominant replacement may take a long period, sometimes up to a couple of minutes for the phenomenon to be seen clearly. For those alarming applications, using EEG channel for detection dozing-off will not be sufficient due to its latency. Slow eye movementlikes other discriminators - is not always precisely related to sleep on-set, however, its appearance is a reliable sign that sleep on-set has happened. In that case, analyzing all three 3 channels to define the transition event is a need.

\section{MATERIALS AND METHODS}

This research applies the Declaration of Helsinki principles in human studies.

\section{Experiment apparatus}

All the experimental process was conducted in Biomedical Laboratory 204B4 at Ho Chi Minh University of Technology. The recording room was designed based on characteristics of Faraday cage in order to reduce the effect of $50 \mathrm{~Hz}$ electrical noise. The data was recorded using NicoletOne V32 device (Natus Neurology Incorporated, WI, USA) providing 32 channels with the ability to amplify the original signal and filter noise at $50 \mathrm{~Hz}$ in order to gain high quality signal. The device is specially designed for sleep analysis and is widely used in many neuroscience laboratories around the world.

Signal gained from NicoletOne device is extracted into digital signal and processed using Matlab software (The Mathworks, Natick, MA, USA).

\section{Technique}

\section{Data acquisition}

Six recordings were collected from volunteered students age ranged from 20 to 25 years old without having neuropathy. Since dozing-off in students happen mostly due to lacking of sleep, all subjects participated in the experiment were asked to reduce the amount of sleep the night before and not to use any alcohol or caffeine before recording. The experiment executed after lunch time 30 to 60 minutes. Before recording, all the objectives and recording process were carefully instructed to subjects.

The data acquisition started with 10-minute period calibration when subject seated, kept their eyes open and relaxed for 5 minutes and thereafter sat with their eyes close for the remaining 5 minutes. The recording in this period was used as training data for later classifying process. During the subsequent 35-minute vigilance test, subject stayed on the same sitting position in front of a screen and a video camera. The task required subjects to watch the display and press a timelapse button whenever they saw a signal (a white $3 \times 1$ centimeters arrow) appeared randomly on the screen. The signal showed up 2 to 4 times per minute and was visible during 4 seconds at each exposure. Any omission of the signal was noticed as a sign of dozing-off event. Data recorded in this period was used as testing data.

\section{Data analysis}

Each signal index required different parameter to examine. The signal processing procedure is shown in Figure 1.

For EEG signal, the predominance of brain waves was calculated based on the Ratio of Power Spectrum (RPS) presented as following formula:

$$
R P S_{x}=\frac{P_{x}}{\Sigma P}
$$

While $\mathrm{X}$ are theta and alpha.

In EMG signal, the reduction in muscle tone was examined by changes in mean square amplitude and identified as:

$$
\overline{A_{E M G}^{2}}=\frac{\sum_{i=1}^{N} A_{i}^{2}}{N}
$$


While N: number of data points in each epoch $A_{i}^{\square}$ : amplitude of each data point $(\mu \mathrm{V})$

EOG channel required a more complicated process to identify slow eye movement feature. By extraction method as executed in previous work ${ }^{6}$, epoch containing SEM was classified automatically under the support of Support Vector Machines algorithm ${ }^{7}$.

First of all, the efficiency of SEM recognition algorithm was evaluated in order to ensure EOG signal analyzing result contribute to the determination of sleep on-set.

After classifying using Support Vector Machines algorithm, epochs with or without SEM appearance were then confronted with the categorized result for each epoch conducted by observation in EEG Viewer software. Epochs were arranged in 4 groups: TP (True Positive) for epochs that were both calculated and observed with SEM; TN (True Negative) for epochs that were both calculated and observed without SEM (non-SEM or NSEM); FP (False Positive) for epochs observed as NSEM but got calculated result as SEM; and FN (False Negative) for epochs observed as SEM but calculated result was NSEM.

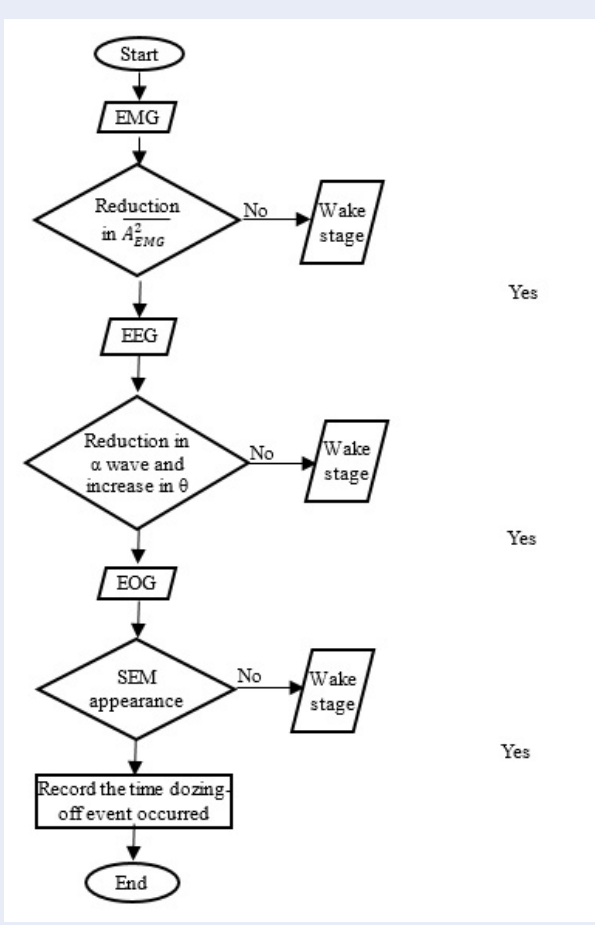

Figure 1: The whole data analysis process was conducted orderly as the following.

The effectiveness of SVM classification algorithm is evaluated based on the following parameters:

$$
\text { Accuracy }=\frac{T P+T N}{\text { total number of epochs }} \times 100
$$

Accuracy gave the general estimation for classifying algorithm.

$$
\text { Sensitivity }=\frac{T P}{T P+F N} \times 100
$$

Sensitivity showed the ability to classify epochs with SEM.

$$
\text { Specificity }=\frac{T N}{T N+F P} \times 100
$$

Specificity showed the ability to recognize epochs without SEM.

Ideally, all three parameters needed to be high to prove the effectiveness of the dozing-off event recognition algorithm. There were two parameters that needed to be noticed: sensitivity and specificity. High sensitivity indicated the optimal effect of SEM recognition algorithm. However, in a successive epoch sequence with SEM, some epoch might be missed and would not change its contribution in detecting dozing-off event but few seconds delay of recognizing the event. Specificity identified NSEM epoch. If the parameter was high, it meant that the errors in identifying the time of sleep on-set due to false detection of slow eye movement was minimized.

\section{RESULTS AND DISCUSSION}

Epoch classification results with NSEM and SEM were listed in Table 1 based on the mentioned parameters: accuracy, sensitivity, specificity.

Table 1: Estimation of SEM classifying algorithm in EOG channel.

\begin{tabular}{llll}
\hline $\begin{array}{l}\text { Sample } \\
\text { number }\end{array}$ & $\begin{array}{l}\text { Accuracy } \\
(\%)\end{array}$ & $\begin{array}{l}\text { Sensitivity } \\
(\%)\end{array}$ & $\begin{array}{l}\text { Specificity } \\
(\%)\end{array}$ \\
1 & 93.43 & 66.67 & 94.61 \\
2 & 96.74 & 66.67 & 98.84 \\
3 & 93.70 & 76.47 & 94.86 \\
4 & 96.43 & 54.55 & 98.14 \\
5 & 82.09 & 62.27 & 89.19 \\
6 & 91.82 & 18.75 & 97.55 \\
\hline
\end{tabular}

According to the results table, the reason of the percentage of sensitivity quite low compared to the percentage of accuracy and specificity is that SEM does not occur alone but rather in a series of repetitive cycles. If the algorithm did not identify all SEMs correctly but correctly identified the first SEMs, this study could identify the time of drowsiness. So as a result, we did not add additional training steps to increase the percentage of sensitivity. 
From the analysis of samples 2 and 3 , it is not possible to use only the EEG signal to determine the state of sleep due to abnormalities occurring in brain waves. Besides, it is not correct to consider chin EMG alone, because this factor has a variable amplitude even in the waking period (sample 3). In the EOG signal, SEM appears in both phases: drowsiness and N1 stage of sleep. Because of these inadequacies, it is necessary to combine many factors to get more accurate and appropriate results in the case of wanting to determine the time of the subject's drowsiness events to monitoring or treatment.

In addition, the time of drowsiness will be detected more slowly due to continuously changing brain wave signals, which makes the state of drowsiness and alertness interwoven in experiments of samples 1 and 4 . It also means that with doze-off alert devices, it is more effective to analyze EMG and EOG signals before investigating the EEG signal when the object is working continuously and having risk of drowsiness. This is very logical because when you fall into a state of muscle tone loss and eye is no longer under control, the accident may occur. Therefore, through the above results, depending on the purpose of the application, consideration can be given to selecting specific signals for analysis and identification.

With the results of the study, it is possible to open a wide range of applications: an alert device for drowsiness or a warning of the imminent drowsiness trend. When early warning is required, priority can be given to EMG and EOG signals. However, when it is necessary to accurately and quantitatively identify trends and the time when drowsiness occurs, an EEG signal analysis can be used.

The suspected dozing-off events were re-checked with the video recorded from camera to determine the real events. The events detected by algorithm were then demonstrated in the same time axis with real events to evaluate the accuracy of the method. There was a total of 11 dozing-off events happened in 6 recordings and 9 events were recognized by algorithms with mean delay time was approximately 50 seconds. Maximum delay time was 2 minutes 20 seconds caused by the steady changes in EEG signal (Figure 2).

Reverse event was observed in a female subject. During the time sleep occurred, the alpha wave took the predominance instead of theta wave (Figure 3). This was an obvious evidence that EEG signal alone could not use to efficiently detect the phenomenon.

\section{CONCLUSIONS}

As can be seen in the result, recording of brain waves only could not be used to determine the state of sleep due to an abnormality might occur in the subject's brainwaves. Also, it is not possible to consider only the muscle tone of the chin because this factor has a variable amplitude. In EOG signal, slow eye movement, as mentioned, appeared in both stages: drowsiness and the N1 stage of sleep. Because of the above inadequacies, the needs of combining all the factors to bring more accurate and optimal results when recording dozing-off events for the purpose of diagnosis and treatment were huge.

Additionally, the phenomenon was more difficult to be detected if the state of sleeping and waking mixed together. When the subject sleepily did a particular action during the experiment, it led to mixing signals in brainwaves. This might force an exclusion of EEG signal and considering EOG and EMG signals instead for a more accurate notification in dozing-off warning device. This was also reasonable since once the muscles lost its ability to stress and eyes was uncontrollable, accidents increased their chances to happen. To conclude, depending on the purpose of the application, consideration of the specific signals may be taken into account for analysis and identification.

\section{ACKNOWLEDGMENT}

This research is funded by Vietnam National University Ho Chi Minh City (VNU-HCM) under grant number C2020-20-10.

\section{LIST OF ABBREVIATION}

EEG: Electroencephalogram.

EMG: electromyogram.

EOG: electrooculogram.

SEM: Slow Eye Movement.

REM: Rapid Eye Movement.

NREM: Non-Rapid Eye Movement.

NSEM: Non- Slow Eye Movement.

\section{AUTHOR S' CONTRIBUTIONS}

All authors contributed equally to this work. All authors have read and agreed to the published version of the manuscript.

\section{CONFLICT OF INTEREST}

We declare that there is no conflict of whatsoever involved in publishing this research. 
(a)

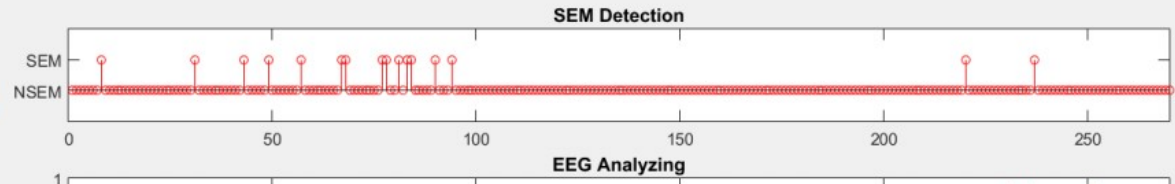

(b)

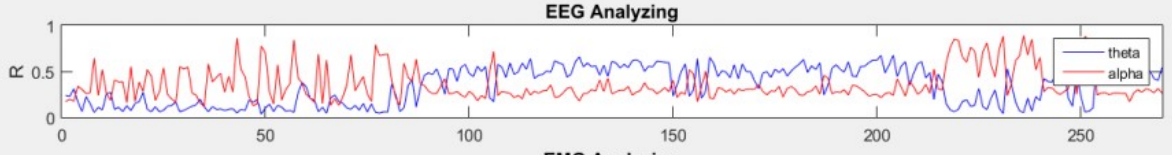

(c)

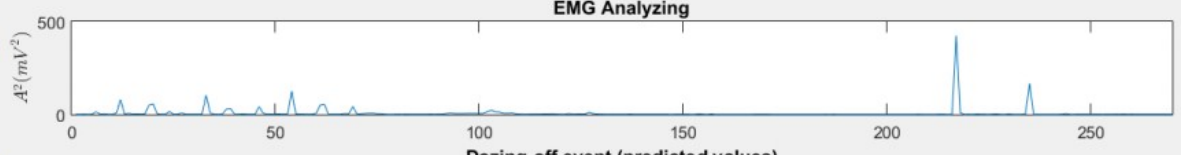

(d)

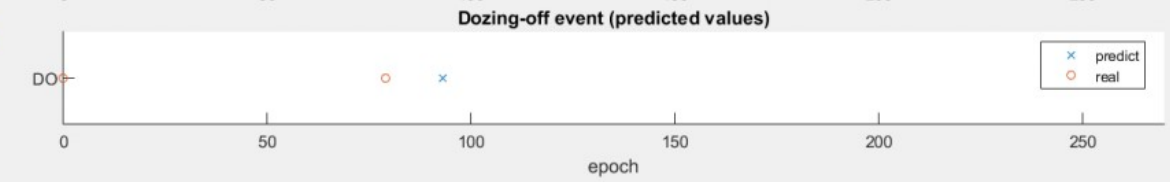

Figure 2: Data analyzing result in EOG, EEG and EMG signals respectively along with dozing-off events marked by algorithm and real events. At the time when sleep on-set occurred, there were the appearance of SEM and reduction in muscle tone. However, the predominance of alpha wave replacing theta wave came after quite a period of time leading to delay in dozing-off recognition.

(a)

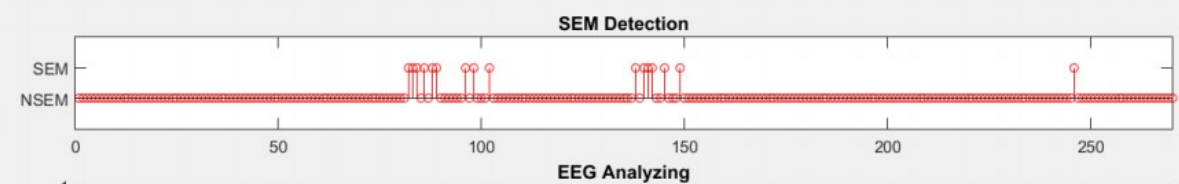

(b)

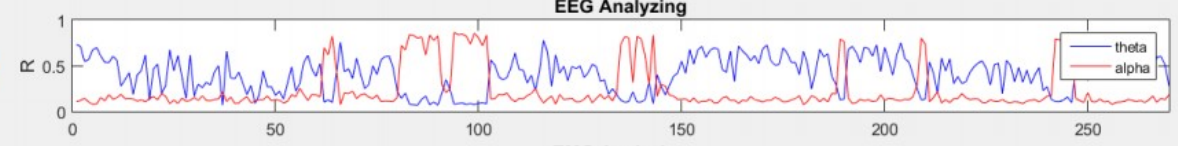

(c)

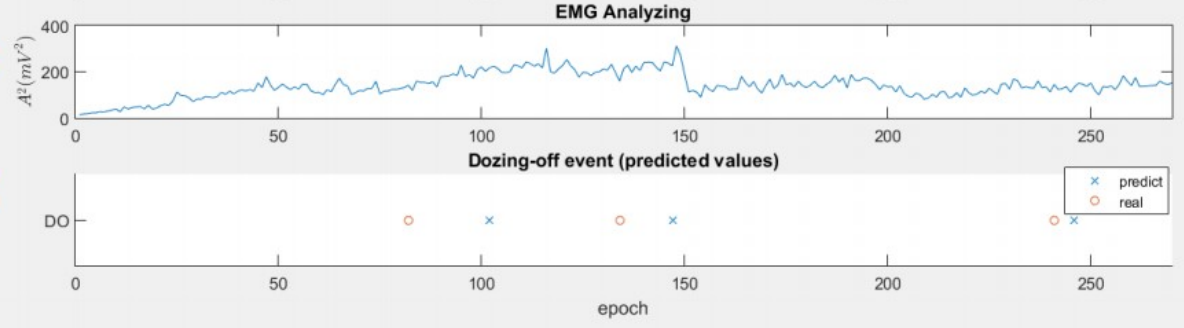

Figure 3: Data analyzing result in EOG, EEG and EMG signals respectively along with dozing-off events marked by algorithm and real events. During sleeping period, subject showed a reverse phenomenon comparing to normal: alpha wave accounted for most of the time while theta wave had less predominance. This event reduplicated when doing another attempt to the same subject with similar conditions.

\section{REFERENCES}

1. Chokroverty S. Overview of sleep \& Sleep disorders. Indian J Med Res. 2010;131(2):126-140.

2. Susman E. Daytime naps maybe a risk factor for a stroke. Neurology Today. 2008;p. 13-15. Available from: https://doi.org/10. 1097/01.NT.0000319946.26513.47.

3. Greenberg MBG. New Study Finds One in Three Teens Are Driving While Drowsy. Liberty Mutual Insurance, Boston. 2016.

4. Dinh TQ, Quang ND. Hệ thống phát hiện tình trạng ngủ gật của lái xe. Tạp chí Khoa học Trường Đại học Cần Thơ, Số chuyên đề: Công nghệ Thông tin (2015). 2015;p. 160-167.
5. Ogilvie RD, McDonagh DM, Stone SN, Wilkinson RT. Eye Movements and the Detection of Sleep Onset. Psychophysiology. 1988;25(1):81-91. PMID: 3353488. Available from: https://doi. org/10.1111/j.1469-8986.1988.tb00963.x.

6. Trang PTH., Hieu NT, Khai LQ, Linh HQ. Detection of Slow Eye Movement in dozing-off event using morphological and Neural Network Method. SEATUC Symposium 2019, Ha Noi, Vietnam. 2019;.

7. Shin D, Sakai H, Uchiyama Y. Slow eye movement detection can prevent sleep-related accidents effectively in a simulated driving task. Journal of Sleep Research. 2010;20(3):416-424. PMID: 21070424. Available from: https://doi.org/10.1111/j.1365-2869. 2010.00891.x. 